\title{
Der Mythos der Drohkulisse. Eine diskursanalytische Untersuchung der Instrumente Baugebot und städtebauliche Enteignung in der responsiven Bodenpolitik
}

\section{The myth of responsiveness. Discourse analysis on the indirect effectiveness of building orders and planning-law expropriations}

https://doi.org/10.2478/rara-2020-0031

Eingegangen: 2. April 2020, Angenommen: 13. Oktober 2020

Kurzfassung: Als besonders eingriffsintensive Instrumente des Baugesetzbuches gelten das Baugebot und die städtebauliche Enteignung. Neben dieser hohen Eingriffsintensität haben beide Instrumente gemeinsam, dass sie selten tatsächlich angewandt werden. Denn die Theorie der responsiven Bodenpolitik besagt, dass zunächst eingriffsärmere Instrumente angewendet werden sollen. Diese Instrumente wirken aber bereits deshalb, weil sich alle Beteiligten und insbesondere die Betroffenen der Existenz eingriffsintensiverer Instrumente bewusst sind. Kommunale Entscheidungsstellen profitieren daher davon, dass das Baugebot und insbesondere die Enteignung als ,ultima ratio' planungsrechtlich vorgesehen ist. Die Responsivität ist gleichwohl nur möglich, wenn die Betroffenen von der Existenz der eingriffsintensiven Instrumente wissen bzw. von ihr erfahren. In diesem Beitrag werden die Ergebnisse einer diskursanalytischen Untersuchung von 300 Zeitungsartikeln der vergangenen 25 Jahre vorgestellt. Der Analyse liegt die Hypothese zugrunde, dass die entscheidenden Ämter in diesen Medien mit der Erwähnung der Instrumente Baugebot und städtebauliche Enteignung bereits im Vorfeld indirekt Einfluss nehmen wollen und zur Kooperation zu überzeugen versuchen. Die Analyse zeigt, dass die Kommunen dazu auch die lokalen Medien nutzen und sowohl das Baugebot als auch die Enteignung in Erwägung ziehen. Häufig wird die tatsächliche Anwendung der Instrumente noch im selben Artikel durch andere Entscheidungsträger/-innen (beispielsweise aus der Opposition), unabhängige Expertinnen/Experten oder die Autorinnen/Autoren des Zeitungsartikels relativiert.

Schlüsselwörter: Wohnungspolitik, Responsive Bodenpolitik, Baugebot, städtebauliche Enteignung, Diskursanalyse, Planungsrecht

\footnotetext{
*Corresponding author: Dr. Michael Kolocek, Technische Universität Dortmund, August-Schmidt-Straße 10, 44221 Dortmund, Deutschland, E-Mail: michael.kolocek@tu-dortmund.de; ILS - Institut für Landes- und Stadtentwicklungsforschung, Brüderweg 22-24, 44135 Dortmund, Deutschland, E-Mail: michael.kolocek@ils-forschung.de

Dr. Andreas Hengstermann, Universität Bern, Hallerstraße 12, 3012 Bern, Schweiz, E-Mail: andreas.hengstermann@giub.unibe.ch, ORCID: 0000-0002-2200-274X
} 
Abstract: Building orders and planning-law expropriations are viewed as particularly interventionist instruments of the Federal Building Code. The two instruments share another characteristic in addition to this high degree of interventionism, namely that they are rarely used in practice. This is due to the theory of responsive land policy, which suggests that less interventionist instruments should be applied first. However, these latter instruments are effective because all those involved, and particularly the affected parties, are aware of the existence of the more intensive options. Municipal decision-making instances thus profit from the provision of building orders and, in particular, expropriation as the 'ultima ratio' of planning law. The responsive approach is nonetheless only possible when those affected are aware of, or are made aware of, the existence of the more interventionist instruments. This article presents the results of a discourse analysis of 300 newspapers from the past 25 years. The analysis investigates the hypothesis that the decision-making instances mention building orders and expropriation in these media so as to exercise indirect influence in advance, and thus attempt to persuade those involved to cooperate. The analysis shows that the municipalities also use the local media to consider the use of building orders and expropriation. Often the actual application of the instruments is relativised in the same article by other decisions (for instance by the opposition), independent experts or editorial comments.

Keywords: Housing Policy, Responsive land policy, Building order, Urban expropriation, Discourse analysis, Planning law

\section{Boris Palmer und die Raumplanung}

Im April 2019 haben rund 240 Grundstückseigentümer ${ }^{1}$ aus der baden-württembergischen Stadt Tübingen einen Brief von ihrem Oberbürgermeister Boris Palmer (Die Grünen) erhalten (o. V. 2019). In Tübingen leben zirka 90.000 Menschen und der Wohnungsmarkt ist, wie in vielen anderen deutschen Städten auch, angespannt. Eine Ursache für den Wohnraummangel ist die fehlende Verfügbarkeit von baureifen Grundstücken. Boris Palmer forderte in diesem Brief die Eigentümer von unbebauten Grundstücken dazu auf, diese zu bebauen oder zum Verkauf freizugeben (o. V. 2019). Andernfalls drohte er innen mit dem Baugebot oder einer Enteignung. Der Brief hat bundesweit für Schlagzeilen gesorgt und insbesondere dem Instrument Baugebot zu einer großen Aufmerksamkeit verholfen.

Bedingt durch die aktuelle Knappheit an bezahlbarem Wohnraum in vielen deutschen Städten scheint die deutsche Bodenpolitik nach jahrzehntelanger Ruhe wieder in Bewegung zu sein. Bodenpolitische Instrumente werden neuerdings intensiv politisch und akademisch diskutiert. Der Städte- und Gemeindebund Nordrhein-Westfalen sammelt erfolgreiche Umsetzun-

1 Grundstücke befinden sich nicht nur im Eigentum von männlichen und weiblichen Privatpersonen, sondern auch im Besitz von Firmen, Banken, Investoren, Immobiliengesellschaften, Familienverbänden etc., deshalb wird in diesem Beitrag das generische Maskulinum „Eigentümer“ verwendet. gen von Baugeboten ${ }^{2}$ und die vom Bundesinnenministerium beauftragte Baulandkommission schlägt unter anderem die Weiterentwicklung von Vorkaufsrechten und Baugeboten im Planungsrecht vor (BMI 2019: 7; vgl. auch Vogel 2019: 27 ff). Selbst das Instrument der Enteignung hat durch die Berliner Initiative zur Enteignung des Wohnungsunternehmens Deutsche Wohnen eine Enttabuisierung erfahren, wenngleich es sich nicht um die städtebauliche Enteignung, sondern um die verfassungsrechtliche Vergesellschaftung handelt (Artikel 15 $\mathrm{GG}^{3}$; vgl. Thiel 2019).

Im vorliegenden Beitrag wird eine Untersuchung des Baugebots ( $\S 176$ BauGB $)^{4}$ und der städtebaulichen Enteignung (§§ 85-122 BauGB) vorgestellt, allerdings aus einer für die Raumplanung eher ungewohnten Perspektive. Sowohl das Baugebot als auch die städtebauliche Enteignung sind bodenpolitische Instrumente (vgl. Hengstermann/Gerber 2015; Hengstermann/Hartmann 2018), da sie neben der nutzungsrechtlichen auch die verfügungsrechtliche Ebene betrachten und darauf abzielen, die beiden Ebenen gegenseitig anzupassen, um so die Inkohärenzen des institutionellen Bodenregimes zu verringern (Gerber/Knoepfel/Nahrath et al. 2009; Viallon/

2 Mitteilung vom 25. Februar 2020, Az.: 20.1.11-020/002.

3 Grundgesetz für die Bundesrepublik Deutschland in der im Bundesgesetzblatt Teil III, Gliederungsnummer 100-1, veröffentlichten bereinigten Fassung, das zuletzt durch Artikel 1 des Gesetzes vom 15. November 2019 geändert worden ist.

4 Baugesetzbuch in der Fassung der Bekanntmachung vom 3. November 2017, das zuletzt durch Artikel 2 des Gesetzes vom 8. August 2020 geändert worden ist. 
Schweizer/Varone 2019). Beide Instrumente stellen weitreichende Eingriffe in die Eigentumsfreiheit dar. Boris Palmers Brief war der Anlass für die vorliegende Untersuchung. Der Beitrag folgt der Ausgangshypothese, dass Entscheidungsstellen in den Kommunen regionale und lokale Medien nutzen, um indirekt Druck auf Eigentümer auszuüben und so eine Handlung bei innen zu erwirken. Allerdings ist nicht das Ziel, einem konkreten Akteur (wie etwa Boris Palmer) eine Diskursstrategie nachzuweisen oder diese zu bewerten. Im Fokus des Interesses stehen die Berichterstattung von lokalen und regionalen Medien und die Suche nach Indizien einer Drohkulisse. Daraus leiten sich folgende Untersuchungsfragen ab:

- Wie häufig berichten regionale oder lokale Zeitungen, dass das Instrument Baugebot bzw. städtebauliche Enteignung angewandt wird oder wurde?

- Wie häufig wird die Anwendung des jeweiligen Instruments angekündigt bzw. angedroht?

- Als wie wahrscheinlich wird die erfolgreiche Umsetzung des jeweiligen Instruments in den Zeitungsartikeln dargestellt?

- Wiereagierendiebetroffenen Grundstückseigentümer oder andere zitierte Akteure?

- In welchen thematischen Zusammenhängen werden die Instrumente erwähnt (Wohnen, Gewerbe, Infrastruktur, Bauruinen, Baulücken, Spekulation)?

Die übergeordnete Frage lautet, ob und wie die entscheidenden Ämter lokale und regionale Medien nutzen, um direkt oder indirekt Drohungen auszusprechen. Der Beitrag betrachtet in Kapitel 2 zunächst die Raumplanung im Spannungsfeld von Macht und Diskursstrategien. In Kapitel 3 werden die Instrumente Baugebot und städtebauliche Enteignung kurz skizziert und hinsichtlich ihrer Eingriffsintensität eingeordnet. In Kapitel 4 folgt eine kurze Beschreibung des Materials und der methodischen Vorgehensweise. Die daran anschließende Ergebnispräsentation in Kapitel 5 bildet die empirische Grundlage für das Fazit (Kapitel 6) zur Wirksamkeit der Responsivität.

\section{Planung, Macht und Diskursstrategien}

Von einigen Ausnahmen abgesehen werden Drohungen entweder mündlich oder schriftlich ausgesprochen. Boris Palmer hat die Eigentümer in Tübingen direkt mit einem Brief angeschrieben und sein Handeln zusätzlich sehr offensiv in der Presse kommuniziert. Neben direk- ten Drohungen gibt es auch indirekte Drohungen. Diese werden verstanden als Andeutungen, häufig verbunden mit Konjunktiven oder Anspielungen: „Wenn X nicht passiert, könnte ich mir vorstellen, dass“ oder "Wir werden prüfen, ob Maßnahme XY möglich ist". Die hohe Anzahl der verschiedenen und zum Teil nicht öffentlichen Diskursebenen ist ein Grund, warum eine erfolgreiche Drohkulisse schwierig nachzuweisen ist.

Die Kommunikation zwischen den Planungsstellen auf der einen Seite und Betroffenen auf der anderen Seite ist planungswissenschaftlich ausführlich erforscht. Einige Forschungsstränge befassen sich mit den formellen und informellen Instrumenten der Partizipation (Bischoff/Selle/Sinning 2007; Selle 2013). Andere untersuchen das Verhältnis von Planung und Macht (Forester 1989; Reuter 2000; Günzel 2016). Healy (1992) hat mit dem "communicative turn" eine breite Diskussion um die Potenziale und Grenzen von Beteiligung und Kommunikation hervorgerufen. In solchen Diskussionen steht allerdings die direkte Kommunikation der Entscheidungsstellen mit den Betroffenen und weniger die indirekte Kommunikation durch die Medien im Vordergrund. Forester (1989: 33 ff.) weist darauf hin, dass auch gezielte Fehlinformationen als strategisches Mittel der Planung eingesetzt werden können. Flyvbjerg (1998) zeigt in seiner detaillierten Fallstudie zu einem großen Stadtumbauprojekt im dänischen Aalborg, wie sich im mehrjährigen Verlauf des Projekts zahlreiche machtvolle Einzelinteressen auf Kosten der besseren Argumentation sowie des Gemeinwohls durchsetzen konnten. Bereits im Jahr 2000 und damit viele Jahre vor Beginn der deutschlandweiten medialen Aufmerksamkeit für "Stuttgart 21“ erläutert Reuter (2000), wie bei den Umbauplanungen des Stuttgarter Bahnhofs der Akteur Deutsche Bahn seine Interessen erfolgreich gegen andere, vermeintlich schwächere Akteure durchsetzen konnte. Hinsichtlich der Bahnhofsplanungen in Stuttgart stellt Reuter fest: „[D]as geäußerte Argument allein genügt nicht, Vortragender, Adressat und Öffentlichkeit spielen ebenso eine Rolle für die Durchsetzung“ (Reuter 2000: 8). Reuter unterscheidet bei der Untersuchung der „Planungspraxis“ zwischen diskursiven Akten und Akten der Macht (Reuter 2000: 10 f.). Alle Äußerungen, die im Verlauf des Planungsprozesses gemacht werden, sind diskursive Akte. Dem gegenübergestellt sind die Akte der Macht, die Reuter (2000: 10) als „alle Möglichkeiten, auf denen die Chance zur Durchsetzung des Interesses beruht", bezeichnet. Die Nutzung von Medien sowie die Einschüchterung betrachtet Reuter als Akte der Macht. An der Einordnung wird sichtbar, dass diskursive Akte und Machtakte häufig nicht klar voneinander zu trennen 
sind. Reuter beschreibt diese Überlappung als eine wechselseitige Beziehung.

Die Wechselbeziehungen von Diskurs und Macht werden häufig mit verschiedenen Methoden der Diskursanalyse eingehend beschrieben. Die Diskursanalyse untersucht Sprache als ein Zeichensystem, mit dem Akteure Bedeutungen produzieren (Keller 2011; Kolocek 2014; Kolocek 2017). Es wird davon ausgegangen, dass hinter dem Gesagten ein Motiv steckt und die systematische Analyse solcher Aussageereignisse auf eine Diskursstrategie hinweisen kann (Schwab-Trapp 2001; Schwab-Trapp 2003; Kolocek 2009). Eine Untersuchung der Diskursstrategien der beteiligten Akteure bei den Planungen von Shopping-Centern hat beispielsweise gezeigt, dass vor allem Projektentwickler (Developer) die erfolgreichste Diskursstrategie hatten und sich gegenüber den Gegnerinnen/Gegnern eines ShoppingCenters sowie den entscheidungstragenden Ämtern mit ihren Interessen häufig durchsetzen konnten (Kolocek 2009). Entscheidungsträger/-innen zeichneten sich vor allem dadurch aus, dass sie sich gegenseitig die Autorität untergruben. Sie schreckten im medialen Diskurs nicht davor zurück, Amtskolleginnen/-kollegen aus der Nachbargemeinde oder einer anderen Partei sehr offensiv anzugreifen, wenn sie mit deren Planungen nicht einverstanden waren, während die Developer konfliktträchtige Themenfelder mieden und sich so geschickt aus der Schusslinie ziehen konnten (Kolocek 2009: 105 ff.).

\section{Instrumente des \\ Baugesetzbuchs und responsive Bodenpolitik}

In diesem Beitrag wird untersucht, ob und wie die Kommunen die Begriffe Baugebot (Kapitel 3.1) und Enteignung (Kapitel 3.2) diskursstrategisch einsetzen. Beide Instrumente werden nachfolgend kurz vorgestellt. Die Theorie der responsiven Bodenpolitik (Kapitel 3.3) erklärt, warum die Instrumente eine Daseinsberechtigung haben, obwohl sie kaum angewendet werden.

\subsection{Das Baugebot (§ 176 BauGB)}

Das Baugebot ist als Instrument des besonderen Städtebaurechts seit $1971 \mathrm{im}$ deutschen Planungsrecht enthalten und im zweiten Kapitel, sechster Teil des Baugesetzbuchs in der Liste der städtebaulichen Gebote (§§ 176-179 BauGB) geregelt (insbesondere in $\S 176$ BauGB). Es ist grundsätzlich als Staatsintervention zu verstehen, die den Bodeneigentümer hoheitlich verpflichtet, ein Grundstück innerhalb einer bestimmten Frist einer bestimmten baulichen Nutzung zuzuführen (Hengstermann/Gerber 2017: 17). Insbesondere die Schließung von Baulücken soll mittels des Baugebots erreicht werden ( $\$ 176$ Abs. 2 BauGB). Gebote stellen per se ungewöhnliche und weitreichende Eingriffe in die Eigentumsfreiheit dar (Müller 1989). Sie entsprechen nicht der sonst üblichen Form von Nutzungsrechten oder -verboten, sondern sind auferlegte Handlungspflichten. Sie sind weitreichend, weil die Wahl über Nutzen oder Nicht-Nutzen des Eigentums aus der Entscheidungsfreiheit des Eigentümers genommen und staatlich getroffen wird. Hinter der städtebaulichen Enteignung ist das Baugebot das bodenpolitische Instrument im deutschen Planungsrecht, welches am weitesten in die Rechte der Privateigentümer eingreift.

Dem Gesetzestext folgend dient das Baugebot dazu, dass eine Gemeinde einem Eigentümer per Bescheid eine Frist setzen kann, bis zu welcher das Grundstück den Bestimmungen im Bebauungsplan anzupassen ist (§ 176 Abs. 1 BauGB). Die Anpassung kann dabei entweder konkret in der Überbauung bestehen (§ 176 Abs. $1 \mathrm{Nr} .1$ BauGB) oder allgemein in einer Anpassung an die baurechtlichen Bestimmungen ( $\$ 176$ Abs. 1 Nr. 2 BauGB). Letzterer Fall ist rechtlich nicht abschließend definiert, zielt politisch jedoch auf erheblich unternutzte Grundstücke (sogenannte geringfügige Bebauung).

Der Gesetzgeber hat vier verschiedene Bedingungen genannt, die kumulativ erfüllt sein müssen, damit eine Anwendung des Baugebots zulässig ist. Erstens müssen, wie bei den anderen städtebaulichen Geboten auch, der amtlichen Maßnahme konsultative Bemühungen der Gemeinde vorausgehen ( $\$ 175$ Abs. 1 BauGB). Gemeinden müssen dem Eigentümer die Maßnahme erörtern und auch auf die Durchführung eingehen. Hierbei ist auch explizit auf öffentliche Finanzierungsmöglichkeiten hinzuweisen. Eine hoheitliche Fristsetzung ohne vorherige Kontaktaufnahme ist somit ausgeschlossen. Zweitens muss die Maßnahme städtebaulich erforderlich und dringend sein ( $§ 175$ Abs. 2 BauGB). Die allgemeine städtebauliche Erforderlichkeit (§ 1 Abs. 3 BauGB) planerischer Tätigkeiten wird folglich um die zeitliche Dimension der Dringlichkeit ergänzt. Den Behörden obliegt es zu begründen, dass die Maßnahme keinen Aufschub duldet und eine eilige Erledigung verlangt. In der planerischen Praxis stellt der kausale und zwingende Nachweis von Erforderlichkeit und Dringlichkeit eine große Herausforderung dar. Dem Gesetzestext folgend ist dringender Wohnbedarf der Bevölkerung explizit als legitimer Anwendungsgrund zulässig. 
Dieser muss jedoch eindeutig nachgewiesen werden, eine unbelegte Nennung ist nicht ausreichend. Drittens erstreckt sich die Möglichkeit des Baugebots grundsätzlich auf Gebiete mit einem Bebauungsplan (§ 176 Abs. 1 BauGB). Um den historisch bedingten Eigenheiten des deutschen Planungsrechts gerecht zu werden, wird eine erweiterte Einsatzmöglichkeit für im Zusammenhang bebaute Gebiete (nach § 34 BauGB) nachgeschoben (§ 176 Abs. 2 BauGB). Eine Anwendung außerhalb dieser beiden Gebietskulissen ist jedoch ausgeschlossen. Viertens muss die Maßnahme verhältnismäßig sein, wobei hiermit explizit auch die wirtschaftliche Zumutbarkeit bezogen auf die Eigentümer gemeint ist (§ 176 Abs. 3 BauGB). Ist die Durchsetzung des Baugebots dem Eigentümer wirtschaftlich nicht zuzumuten, ist von der Anwendung des Instruments abzusehen (§ 176 Abs. 3 BauGB) oder er kann die Übernahme des Grundstücks durch die Gemeinde verlangen (§ 176 Abs. 4 BauGB), wobei dann die planungsrechtlichen Entschädigungsregeln zur Anwendung kommen.

Die Einreichung des Antrags auf Baugenehmigung wird dabei nach allgemeiner Rechtsauffassung als Erfüllungstatbestand verstanden. Für den Fall, dass dies nicht innerhalb der gesetzten Frist geschieht, sieht das Baugesetzbuch zwei abgestufte Rechtsfolgen vor ( $§$ 176 Abs. 8 und 9 BauGB). Zunächst wird auf die landesrechtlichen Vollstreckungsmaßnahmen verwiesen, wobei für das Baugebot allein das Zwangsgeld relevant sein dürfte, da andere Zwangsmittel (wie die Erzwingungshaft) wirkungslos und unverhältnismäßig wären. Verschärfend kann dann in einer zweiten Stufe die Enteignung eingeleitet werden ( $§ 85$ Abs. 1 Nr. 5 BauGB). Diese Rechtsfolge ist jedoch folgenlos, da es sich dabei um ein eigenständiges Enteignungsverfahren handelt und keine über das allgemeine und ohnehin geltende Enteignungsrecht hinausgehende Sanktionsmöglichkeit darstellt (§ 176 Abs. 2 S. 2) (Hengstermann 2017: 154 f.). Baugebot und Enteignung sind demnach eigenständige, parallel verlaufende Verfahren und nicht (im Sinne einer responsiven Bodenpolitik) aufeinander abgestimmt. Andere abgestufte Rechtsfolgen, wie beispielsweise in der Schweiz der entschädigungslose Wegfall des Baurechts (z. B. § 36c PBG-SZ ${ }^{5}$ ) oder eine Sonderbesteuerung (z. B. § 28j BauG-AG ${ }^{6}$ ), sind im deutschen Baurecht nicht vorgesehen (Hengstermann 2017: 158).

5 Planungs- und Baugesetz des Kantons Schwyz vom 14. Mai 1987.

6 Gesetz über Raumentwicklung und Bauwesen des Kantons Aargau vom 19. Januar 1993, Stand 1. Januar 2011.
Gesamthaft zeigt sich, dass das Baugebot konzeptionell von hoher Bedeutung für ein konsequentes Planungssystem sein könnte, in der derzeitigen planungsrechtlichen Form jedoch planungspraktisch kaum durchsetzbar ist. Die engen Anwendungsvoraussetzungen (kausale Nachweise der Erforderlichkeit und Dringlichkeit), die hohen Hürden an die Verhältnismäßigkeit (wirtschaftliche Zumutbarkeit für den einzelnen Eigentümer), die mögliche Übernahmepflicht durch die Gemeinden (vor dem Hintergrund der aktuellen wirtschaftlichen Lage der kommunalen Haushalte) und die fehlenden wirksamen Sanktionsmöglichkeiten bei Nichtbeachtung (kein Enteignungsvorgriff) führen dazu, dass das Baugebot nach deutschem Recht als "stumpfes Schwert" (Stüer 1988: 337) oder „unvollkommen“ (Lücke 1980: 37) bezeichnet wird.

Trotz dieser rechtswissenschaftlichen Skepsis wird das Instrument planerisch als wertvoll erachtet (Schlichter 1993: 365 ff.) und regelmäßig als „wirksame Drohkulisse" (Leisner 2009: 22) tituliert oder missbraucht (Leisner 2009: 77 f.). Die Hoffnungen liegen dabei auf der indirekten Wirkung durch den reinen Verweis auf den Gesetzestext (Müller 1989: 180 f.; Hengstermann/Gerber 2017: 18), ungeachtet der tatsächlichen rechtswissenschaftlichen Qualität (Kolocek 2018: 189 ff.).

\subsection{Die städtebauliche Enteignung ( $\S$ 85-122 BauGB)}

Die städtebauliche Enteignung ist seit 1953 im bundesdeutschen Planungsrecht enthalten. Zunächst im Baulandbeschaffungsgesetz zum Zwecke des Wohnungsbaus ist sie seit 1960 im ersten Kapitel, fünfter Teil des damaligen Bundesbaugesetzes und heutigen Baugesetzbuchs ( $\S 85-122$ BauGB) geregelt. Mit der städtebaulichen Enteignung wird der teilweise oder gar vollständige Entzug von Eigentumsrechten an einem bestimmten Grundstück auf der Grundlage des Baugesetzbuchs bezeichnet (Schlick 2018: 115). Die Zustimmung des Eigentümers ist dafür nicht notwendig, wohl aber eine angemessene Entschädigung gemessen am Verkehrswert des Grundstücks (Art. 14 Abs. 3 GG; § 93 BauGB), weshalb die Enteignung auch als Zwangskauf (Lege 2011: 508) oder - in der Schweiz - euphemistisch als hoheitliches Kaufrecht (Art. 126b BauG-BE) ${ }^{7}$ bezeichnet wird. Die Enteignung stellt den weitreichendsten Eingriff in das Privateigentum dar. Die Enteignungsmöglichkeit und die Eigentumsgarantie sind dabei jedoch nicht

7 Baugesetz des Kantons Bern vom 9. Juni 1985. 
als Gegensätze, sondern sich gegenseitig bedingende Eigenschaften zu verstehen.

Dem aktuellen Gesetzestext folgend ist die rechtliche Zulässigkeit einer Enteignung in Deutschland relativ eng gefasst und detailliert geregelt (vgl. Schmidt-Eichstaedt 2005: 403 ff.). Neben der Enteignung als Einzelmaßnahme ( $\S \S 85-122$ BauGB) ist eine Enteignung auch als integrierter Bestandteil einer städtebaulichen Entwicklungsmaßnahme (§§ 165-171 BauGB) oder auf Grundlage anderer Rechtsgebiete (z. B. Bergbau, Eisenbahn- und Straßenbau) möglich. Diese nichtbaurechtlichen Enteignungsmöglichkeiten sind jedoch nicht Gegenstand des vorliegenden Beitrags, wenngleich es in der Politik und Öffentlichkeit nicht selten zu Missverständnissen führt, wie die eingangs erwähnte aktuelle Vergesellschaftungsdebatte in Berlin zeigt.

Die Voraussetzung einer Enteignung ist, dass sie dem Wohl der Allgemeinheit dient und der Enteignungszweck auf andere zumutbare Weise nicht erreicht werden kann (§ 87 Abs. 1 BauGB). Explizit erwähnt wird dabei, dass die Verwaltung sich vordergründig um einen freihändigen Erwerb bemühen muss. Zwar wird dabei im Gesetz auf die angemessenen Bedingungen verwiesen; „praktisch wirkt sich diese Vorschrift jedoch preistreibend aus, da die Verwaltung in der Verhandlungsphase auf das Entgegenkommen des Grundeigentümers angewiesen ist" (Schmidt-Eichstaedt 2005: 405) und auch aus ihrer Sicht der freihändige Erwerb dem Enteignungsverfahren vorzuziehen ist.

Die Anwendung der Enteignung ist auf wenige Zwecke beschränkt. Im Gesetz heißt es allgemein, dass enteignet werden kann, um Grundstücke entsprechend der Festsetzungen des Bebauungsplans zu nutzen oder eine solche Nutzung vorzubereiten ( $\S 85$ Abs. $1 \mathrm{Nr} .1$ BauGB). Die Rechtsprechung konkretisiert diese sehr allgemeine Formulierung dahingehend, dass damit ausschließlich öffentliche Bodennutzungen gemeint sind (vgl. „Boxberg-Urteil“8, auch aufgenommen in § 87 Abs. 3 BauGB). Die Enteignung kann also der Beschaffung von Grundstücken dienen, die zur Erfüllung öffentlicher Aufgaben notwendig sind. Klassische Beispiele sind Flächen für den Bau von technischer oder sozialer Infrastruktur, z. B. Straßen und Schulen.

Daneben enthält der Gesetzestext sechs weitere Zwecke, die in der planerischen Praxis jedoch von deutlich geringerer Bedeutung sind. So ist es möglich, mit der Enteignung Baulücken im Innenbereich (§34 BauGB) zu schließen (Nr. 2) oder Flächen für Entschädigungszwecke (Nr. 3) oder Ersatzleistungen (Nr. 4) zu mobilisieren.

8 BVerfGE 74, 264.
Die Enteignung als Rechtsfolge eines nicht beachteten Baugebots (siehe oben) wird ebenfalls explizit genannt (Nr. 5). In diesem Fall kann die Enteignung ausdrücklich auch zugunsten eines bauwilligen Privaten erfolgen (§ 87 Abs. 3 S. 2 BauGB). Schließlich sind auch Enteignungen im Bereich der Erhaltungs- ( $\S 85 \mathrm{Nr} .6$ BauGB) und seit 2004 auch im Bereich der Stadtumbausatzung (Nr. 7) möglich, wenn eine Zielerreichung auf anderem Wege nicht gewährleistet ist.

Ergänzend zu den Bestimmungen der Enteignung als Einzelmaßnahme ist das Instrument auch Bestandteil der städtebaulichen Entwicklungsmaßnahme (SEM) (§§ 165-171 BauGB). Einem integrierten Planungsansatz folgend soll die städtebauliche Entwicklungsmaßnahme ermöglichen, dass Gemeinden (oder beauftragte Entwicklungsträger) Planvorhaben von besonderer Bedeutung einheitlich und zügig umzusetzen vermögen. Als Besonderheit des Instruments wird die eigentumsrechtliche Dimension der Planung mitgedacht und explizit berücksichtigt. Die Gemeinden agieren dabei grundsätzlich als Zwischenerwerber und sollen die entsprechenden Grundstücke zu angemessenen Bedingungen erwerben (§ 166 Abs. 3 BauGB). Dadurch entsteht ein Verkaufs- oder Kooperationszwang, da für den Fall, dass eine Einigung mit den bisherigen Eigentümern nicht möglich ist, das Gesetz ausdrücklich die Möglichkeit der Enteignung vorsieht (§ 169 Abs. 3 BauGB). Diese enteignungsrechtliche Vorwirkung wirkt dann (im Unterschied zur Enteignung nach $\S \S 85 \mathrm{ff}$ ) auch in Bereichen ohne Bebauungsplan. Der besonderen Bedeutung des Planvorhabens für die jeweilige Gemeinde folgend soll so sichergestellt werden, dass die tatsächliche Umsetzung gewährleistet ist.

Sowohl als Einzelmaßnahme als auch als Teil der städtebaulichen Entwicklungsmaßnahme ist die Enteignung verfassungsrechtlich an die Anforderung geknüpft, dass dabei oder bei enteignungsgleichen Eingriffen in das Eigentum eine Entschädigung erfolgt (Art. 14 Abs. 3 S. 2 GG), um so zumindest eine Wertgarantie zu erhalten. Das Allgemeinwohl ist hier sowohl als Bedingung als auch als Rechtfertigung eines Eingriffs in das Grundrecht auf Eigentum zu verstehen, wobei die Frage „was im öffentlichen Interesse liegt, essentiell politischer Natur [ist], ja der politische Entscheid par excellence. Wandlungen in der Deutung des Begriffs reflektieren stets eine gewandelte Auffassung von den Aufgaben des Staates“" (Saladin 1982: 352).

Gesamthaft betrachtet ergibt sich, dass die städtebauliche Enteignung sowohl als Einzelmaßnahme als auch als Teil der städtebaulichen Entwicklungsmaßnahme ein grundsätzlich anwendbares Instrument des 
Planungssystems ist. Jedoch ist auch dieses Instrument an hohe bürokratische und verfahrensrechtliche Hürden geknüpft und zudem auf eine enge Auswahl an recht genau definierten Fällen begrenzt - insbesondere zur Beschaffung von Grundstücken, die zur Erfüllung öffentlicher Aufgaben notwendig sind. Es dient damit in der Planungspraxis als Spezialinstrument in genau diesen Fällen, eignet sich nach seinem rechtswissenschaftlichen Charakter jedoch nicht als allgemeines, flächendeckendes Instrument, um etwa wohnungspolitische Ziele zu erreichen.

\subsection{Responsive Bodenpolitik}

Die aktuellen bodenpolitischen Debatten kursieren um die Frage, wie die soeben vorgestellten und andere Instrumente strategisch kombiniert und angewandt werden können. Einerseits sollen die Eigentümer aus der VetoPosition der Planumsetzung herausgeholt werden. Andererseits muss eine ausgewogene Anwendung der Instrumente auch einem ausbalancierteren Verhältnis zwischen der Eigentumsgarantie und der Sozialpflichtigkeit des Eigentums (vgl. Prantl 2019; Vogel 2019) Rechnung tragen. In beiden Fällen soll die Planumsetzung gewährleistet und die Erreichung der planungspolitischen Ziele (wie zusätzlicher Wohnungsbau ohne weitere Flächeninanspruchnahme) ermöglicht werden.

Die strategische Kombination der Instrumente beruht auf der Annahme, dass die Instrumente in einer logischen Abfolge entlang der Eingriffsintensität aufeinander aufbauen und konkrete Vorhaben mit dem geringstmöglichen Eingriff realisiert werden sollen. Dafür müssen die eingriffsintensiven Instrumente existieren und deren Anwendbarkeit grundsätzlich rechtlich möglich sein. Die aufeinander reagierende Funktion der Instrumente wird metaphorisch als Umsetzungspyramide illustriert und als responsive Bodenpolitik bezeichnet (Davy 2005: 70). Trotz der Existenz und der Anwendbarkeit liegt in der Responsivität die Annahme, dass die tatsächliche Anwendung der eingriffsintensiven Instrumente gar nicht notwendig sei, um die intendierte Wirkung zu erzielen (Davy 2005: 70 f.; Hengstermann 2017: 153 f.; Hengstermann/Gerber 2017: 17 f.). Strategisch kombiniert führen schon die weniger eingriffsintensiven Instrumente Information und Beratung, moralische Appelle, ökonomische Anreize (Davy 2006: 134) - dazu, dass Lösungen auf geringerer Intensitätsstufe gefunden werden; die eingriffsintensiven Instrumente sollen indirekt wirken.

Ob diese indirekte Wirkung jedoch tatsächlich erreicht wird, ist planungswissenschaftlich und metho- disch schwierig nachzuweisen. Bisherige Untersuchungen fokussieren meist auf spezifische Detailaspekte. So finden sich rechtswissenschaftliche Beurteilungen der Frage, ob einzelne Instrumente verfassungskonform seien und justiziabel anwendbar wären (Köhler 1985; Antoniazza 2008) oder ob die Anwendung im konkreten Fall verhältnismäßig war (Dieterich 1985; Stüer 1988). Ersteres wäre zwar praktisch relevant, basiert jedoch auf Überlegungen, die meist ausschließlich rechtstheoretischer Natur sind und andere, nicht rechtswissenschaftliche Aspekte vernachlässigt. Letzteres basiert auf Gerichtsurteilen - also auf Fällen der tatsächlichen Anwendung inklusive juristischer Eskalation. Rückschlüsse auf die systemische Wirkungsweise der Instrumente oder übertragbare Aussagen für die planerische Praxis lassen sich aus diesen rechtswissenschaftlichen Ansätzen nicht ableiten. Daneben sind lediglich politikoder planungswissenschaftliche Untersuchungen einzelner Fälle (best practice) zu finden (Dieterich 1983; Schäfer 1983; Lobeck/Wiegandt/Wiese-von Ofen 2006; Hengstermann/Gerber 2017).

Solche Forschungsansätze sind daher aufgrund ihrer methodischen Ansätze nicht in der Lage, die Wirkung des angenommenen responsiven Mechanismus nachzuweisen. So bleibt bislang unklar, ob bereits die rechtliche Existenz des Baugebots eine Verflüssigung des Baulandes bewirkt und ob allein die Möglichkeit einer Enteignung zu einer sozialgerechteren Bodennutzung führt. Eine dazu benötigte Verhaltensveränderung der Eigentümer ist „weder Gegenstand von Untersuchungen im Schrifttum noch von Gerichtsentscheiden" (Leisner 2009: 77). Auch der vorliegende Beitrag kann einen solchen Nachweis nicht eindeutig liefern. Gleichwohl zeigt er anhand der Untersuchung von rund 300 ausgewählten Zeitungsartikeln die Zusammenhänge auf, in denen das Baugebot und die städtebauliche Enteignung öffentlich diskutiert werden. Die Untersuchung folgt der Ausgangshypothese, dass entscheidenden Ämter regionale und lokale Medien nutzen, um indirekt Druck auf Eigentümer auszuüben und so eine Handlung bei innen zu erwirken.

\section{Datengrundlage und methodische Vorgehensweise}

Die Datengrundlage besteht aus 301 Artikeln aus regionalen und lokalen Zeitungen, die zwischen 1990 und 2019 in Deutschland publiziert wurden. Die Artikel wurden mithilfe der Datenbank Nexis Uni recherchiert. 
Tabelle 1: Datenkorpus (Anzahl der untersuchten Zeitungsartikel)

\begin{tabular}{lllll}
\hline Thema & $\mathbf{1 9 9 0 - 1 9 9 9}$ & $\mathbf{2 0 0 0 - 2 0 0 9}$ & $\mathbf{2 0 1 0 - 2 0 1 9}$ & Gesamt \\
\hline Ausschließlich Baugebot & 4 & 20 & 92 & $\mathbf{1 1 6}$ \\
Ausschließlich Enteignung & 17 & 57 & 67 & $\mathbf{1 4 1}$ \\
Beide Instrumente & 4 & 7 & 33 & $\mathbf{4 4}$ \\
Gesamt & $\mathbf{2 5}$ & $\mathbf{8 4}$ & $\mathbf{1 9 2}$ & $\mathbf{3 0 1}$ \\
\hline
\end{tabular}

Nexis Uni ist eine Rechercheplattform für Universitäten und Bibliotheken und erlaubt die Recherche unter anderem von Rechtsdaten, Nachrichten und Unternehmensdaten weltweit. Für die Recherche in deutschsprachigen Zeitungen greift die Plattform auf regionale und lokale Zeitungen (z. B. Nürnberger Nachrichten, Rheinische Post) und überregionale Zeitungen (z. B. Die Welt, taz) zurück. Die überregionalen Zeitungen haben häufig eigene Regional- und Lokalteile, die ebenfalls berücksichtigt wurden. Lokale und regionale Zeitungen zeichnen sich dadurch aus, dass sie zwar regelmäßig über nationale und internationale Themen berichten, aber vor allem lokale Planungen und gegebenenfalls die damit einhergehenden Konflikte thematisieren. Da die Kommunikation kommunaler Entscheidungsträger im Interessensfokus lag, wurde dieser Dokumententypus gewählt. Alle Artikel liegen den Autoren digital vor; die überwiegende Anzahl ist in den Printmedien erschienen und wurde nachträglich digitalisiert. Im Rahmen der Recherche zum Baugebot wurden aufgrund der verhältnismäßig niedrigen Trefferquote alle gefundenen Artikel aufgenommen. Bei der Recherche zur städtebaulichen Enteignung wurde für den Zeitraum ab 2010 aufgrund der hohen Trefferquote eine zufallsgenerierte Stichprobe erhoben. Als erstes Ergebnis kann somit festgehalten werden, dass die Enteignung in der medialen Berichterstattung häufiger vorkommt als das Baugebot.

Tabelle 1 zeigt die Anzahl der Artikel verteilt auf drei Zeiträume. 116 Artikel behandeln ausschließlich das Baugebot, 141 Artikel die Enteignung und in 44 Artikeln tauchen beide Instrumente auf. Zwischen 1990 und 1999 konnten insgesamt nur 25 Artikel ausfindig gemacht werden. Aus den Zahlen dürfen keinerlei Schlussfolgerungen hinsichtlich der Entwicklung der Aufmerksamkeit für die beiden Instrumente getroffen werden. Der Grund für eine wachsende Trefferzahl in den späteren Untersuchungsräumen liegt vor allem in dem wachsenden Suchfeld der Rechercheplattform. Für den Zeitraum 1990 bis 1999 hat Nexis Uni noch auf weniger Zeitungsarchive zugreifen können. Aufgrund der geringen Anzahl aus diesem Zeitraum wurde bei der Ergebnisdiskus- sion auf Auswertungen zu zeitlichen Entwicklungen und Unterschieden verzichtet. Das untersuchte Material ist als empirische Grundlage zur Beantwortung der Forschungsfragen hinsichtlich seiner Repräsentativität mit der für quantitative Forschung üblichen Vorsicht einzuschätzen. Eine Erhebung, die alle lokalen und regionalen Zeitungen Deutschlands repräsentiert, war aus verschiedenen Gründen (Datenverfügbarkeit, Forschungsökonomie) nicht möglich, aber auch nicht beabsichtigt.

DieArtikel wurden in das SoftwareprogrammATLAS.ti geladen und zunächst zusammengefasst. In einem weiteren Schritt wurden die Artikel zunächst hinsichtlich des Instruments (Baugebot, Enteignung, beide Instrumente), der Untersuchungsfragen sowie des (groben) thematischen Kontexts (z. B. Wohnen, Gewerbe, Rechtsstreit, Bauruine) codiert. Die meisten Artikel behandeln mehrere Themen gleichzeitig, beispielsweise wenn die Autorin oder der Autor über einen Rechtsstreit im Rahmen der Neuplanungen eines Gewerbegebiets berichtet wurde oder ein neues Gebiet mit Wohn- und Gewerbebebauung entwickelt werden sollte. Daher waren Mehrfachcodierungen einzelner Artikel eher die Regel als die Ausnahme. Die statistische Auswertung der Codes erfolgte über ATLAS.ti und Microsoft Excel.

\section{Ergebnisse}

Im Mittelpunkt der nun folgenden Ergebnisdiskussion stehen die Besonderheiten der jeweiligen Instrumente sowie die wesentlichen Unterschiede. Der erste Teil enthält die Erkenntnisse, die zum Baugebot gewonnen werden konnten, und der zweite Teil bezieht sich auf die städtebauliche Enteignung. Der dritte Teil umfasst die Artikel, die beide Instrumente berücksichtigen.

\subsection{Baugebot}

Von den 301 untersuchten Artikeln befassen sich 116 mit dem Baugebot. Nur acht der 116 Artikel (7\%) the- 
matisieren (scheinbar) die Anordnung eines Baugebots. Ein genauer Blick auf diese acht Artikel macht gleichwohl deutlich, dass es sich in sechs dieser acht Fälle um Begriffsmissverständnisse handelt. Diese Zeitungsartikel berichten nicht über Baugebote nach § 176 BauGB. Stattdessen handelt es sich um vertragliche Vereinbarungen zwischen der Gemeinde und den Bauherren. Die Autorinnen und Autoren benutzen den Begriff „Baugebot", wenn sie darüber berichten, wie sich die neuen Grundstückseigentümer und die Gemeinde vertraglich darauf geeinigt haben, dass ein erworbenes Baugrundstück zeitnah bebaut wird. Wenn eine Gemeinde plant, Neubaugrundstücke mit einer solchen vertraglichen Bauverpflichtung zu versehen, dann wird diese Vereinbarung im Artikel schon mal irrtümlicherweise als Baugebot bezeichnet: „Außerdem gibt es noch vier Plätze im Bereich der Ortserweiterung Wieseck und einen in Petersweiher. Eines haben alle diese Plätze gemeinsam: Sie unterliegen einem Baugebot. Das bedeutet, dass nach dem Kauf innerhalb von zwei Jahren ein bezugsfertiges Haus entstanden sein muss. Nur in gut begründeten Einzelfällen kann diese Frist um einige Monate verlängert werden" (Goltze 2011).

Ein Artikel über ein erfolgreich vollzogenes Baugebot nach § 176 BauGB konnte im Datenkorpus nicht ausfindig gemacht werden. Die taz berichtet in ihrem Regionalteil lediglich über einen Rechtsstreit, den die Anordnung eines Baugebots verursacht hat (von Appen 1994). In einem anderen Fall berichtet die Frankfurter Rundschau, dass dem von der Stadt ausgesprochenen Baugebot von Fachleuten wenig Aussicht auf Erfolg eingeräumt wird (Göpfert 2003). Das Datenmaterial stützt eine Vermutung aus der Fachliteratur: In Deutschland wird das Instrument Baugebot selten formal angeordnet. Ein weiteres Indiz für diese Erkenntnis ist die äußerst niedrige Anzahl der gerichtlichen Entscheidungen zum Baugebot (Wissenschaftliche Dienste des Bundestags 2018: 4).

Im Datenkorpus dominieren Zeitungsartikel, in denen die Möglichkeit eines Baugebots in Erwägung gezogen wird (67 von 118 Artikeln, 58 \%). Hier sind die direkten Androhungen häufiger und deutlicher (Goethals 2007; Stiefelhagen 2011) als in der Berichterstattung zur Enteignung (siehe unten). Häufig handelt es sich dabei um Flächen, die von den Eigentümern vernachlässigt werden: „Bezirksverwatungs-Chef Siegfried Tietzka: ,Bislang konnten wir den Grundstückseigentümer nur per Ordnungsverfügung auffordern, für mehr Sauberkeit zu sorgen. Doch jetzt werden wir mehr Druck machen. Ich werde das Bauaufsichtsamt bitten, ein mögliches Baugebot des Eigentümers zu prüfen. Dann könnte er das Grundstück nicht länger leer stehen und verrotten lassen, sondern müsste eine Bebauung vornehmen"“ (Hille 2000).

Gleichwohl wird die tatsächliche Wahrscheinlichkeit einer Umsetzung hin und wieder noch im selben Artikel relativiert, allerdings in nur 15 von 67 Fällen (22 \%): "Das ganz und gar unfriedliche Mittel eines behördlichen Baugebots sehen die Baulückenspezialisten nur als ,ultima ratio, die sie lieber vermeiden. In der City etwa müssten sie nachweisen, dass - beispielsweise - eine Aufstockung ,objektiv“ wirtschaftlich sei. ,Keine Chance', meinen beide. Also beraten sie lieber, locken mit zinsgünstigen Hypotheken und anderem Zuckerbrot. Und verraten potenziellen Bauherrn, wo es noch schöne Baulücken gibt“" (o. V. 2000).

Ein wesentlicher Unterschied zur Enteignungsberichterstattung ist, dass in den Zeitungen häufiger konkret erklärt wird, woran die Umsetzung scheitert. Es werden juristische Probleme erwähnt (Biermann 2007) oder mehr oder weniger direkt auf die finanziellen Hürden (o. V. 2004a) hingewiesen. Ein Beispiel: „Zwar hätte die Hotaka [Hotel am Kaiserdamm $\mathrm{GmbH}$ ] mit einem Baugebot zur Fertigstellung gezwungen werden können. Doch im Falle der Verweigerung seitens des Bauherren wäre der Bezirk in die Pflicht gekommen. ,Wir hätten Grundstück und Rohbau kaufen und fertig stellen müssen.' Doch angesichts leerer Kassen sei dies unmöglich. Daher hatte Gröhler, noch als Wirtschaftsstadtrat, im August [den Geschäftsführer des Eigentümers] nur um eine Stellungnahme ersucht" (o. V. 2001).

Die Sächsische Zeitung (Wolf 2016) bezeichnet das Baugebot als einen "Papiertiger" und andere Zeitungen, wie z. B. der Kölner Stadt-Anzeiger und der Gießener Anzeiger, greifen das Bild des "stumpfen Schwerts" (Puderbach 2012; o. V. 2016) auf. 29 Artikel (25\%) fallen dadurch auf, dass das Instrument zwar erwähnt, aber schon ausgeschlossen wird, bevor die Leser/-innen überhaupt ernsthaft mit einer tatsächlichen Anwendung rechnen können (Göpfert 2003; o. V. 2017a). Hier deutet manchmal schon die Überschrift auf eine niedrige Umsetzungswahrscheinlichkeit hin, wie zum Beispiel in den Nürnberger Nachrichten vom 17. März 2015: „Stadt hat nur ein stumpfes Schwert. Zwang hilft beim Kaufhof nicht" (o. V. 2015). Die Stuttgarter Nachrichten berichten über das Ziel der Stadt, neue Wohneinheiten schaffen zu wollen. Im Artikel werden die politisch Agierenden verschiedener Parteien mit unterschiedlichen Vorschlägen (z. B. Umlegungsverfahren, Flächenkauf) zitiert. Auch die Schließung von Baulücken wird als eine Option betrachtet: „[Der Stadtkämmerer] pocht auf das Schließen von Baulücken. [Der Leiter des Amtes für Liegenschaften 
und Wohnen] Zügel hat dabei ernüchternde Erfahrungen gemacht. Anschreiben hätten die meisten Besitzer ignoriert. ,Die Aktion bringt nur einen Tropfen auf den heißen Stein, wir machen dennoch weiter', sagt er. Dass der Gemeinderat sich dazu entschließen könnte, einen Bauzwang für die wertvollen Baulücken zu verhängen, sei kaum zu erwarten. Zügel: ,Für ein Baugebot müssten die Zeiten wohl noch ganz anders werden." (Schwarz 2005).

Die thematische Breite ist in den Artikeln zum Baugebot nicht ganz so groß wie bei der Enteignung (siehe unten). Gewerbeplanungen spielen nur eine geringe Rolle (16 Artikel, $14 \%$ ). Die thematischen Schwerpunkte sind Wohnen (57 Artikel, $49 \%$ ) und Bauruinen (47 Artikel, $41 \%)$. In vielen Artikeln werden beide Themen behandelt. Im Jahr 2014 berichtet der Gießener Anzeiger über eine seit über 20 Jahren leerstehende Bauruine „An der alten Post“. Diese solle für Wohnzwecke verfügbar gemacht werden. Auch hier endet der Artikel allerdings mit dem Hinweis, dass ein Baugebot unwahrscheinlich erscheint: „Zwischenzeitlich hatten Vertreter von SPD und Grünen das Instrument eines Baugebots für das ehemalige Postgebäude ins Spiel gebracht. Hierbei kann die Stadt den Eigentümer verpflichten, innerhalb einer zu bestimmenden Frist ein vorhandenes Gebäude entsprechend der Festsetzungen des Bebauungsplans anzupassen. Der Knackpunkt hierbei: Für das Areal um die Alte Post gibt es keinen gültigen Bebauungsplan“ (o. V. 2014a).

Im Gesamtbild entsteht jedoch bei der Berichterstattung zum Baugebot kein Eindruck, der bei zeitunglesenden Eigentümern eine Änderung des Verhaltens bewirken würde.

\subsection{Enteignung}

Insgesamt befassen sich 141 Zeitungsartikel aus dem Datenkorpus ausschließlich mit der städtebaulichen Enteignung. Zehn der 141 Artikel (7\%) berichten, dass eine Enteignung tatsächlich angeordnet oder schon durchgeführt wurde. In allen diesen zehn Artikeln wird von einem Rechtsstreit berichtet, entweder über die Zulässigkeit des Enteignungsbeschlusses (Lauchs 1994) oder über die Höhe der Entschädigung: „Es gibt noch Forderungen von Grundstückseigentümern, die nach ihrer Ansicht damals nicht angemessen für Enteignungen entschädigt worden sind. Das Oberlandesgericht Frankfurt hatte im März 2007 fünf Klägern Recht gegeben und die Stadt zur Zahlung von knapp drei Millionen Euro verpflichtet" (o. V. 2009a).
Wenn eine Zeitung über eine angeordnete oder durchgeführte Enteignung berichtet, dann nie ohne zu erwähnen, dass sich die betroffenen Eigentümer zur Wehr setzen. Eine mögliche Erklärung könnte sein, dass die Anordnung einer Enteignung nur dann als erwähnenswert erscheint, wenn es zum Rechtsstreit kommt und in anderen Enteignungsfällen einfach nicht medial berichtet wird.

In 52 der 141 Artikel (37\%) steht, dass die Enteignung zwar (noch) nicht angeordnet wird, aber die Möglichkeit in Aussicht gestellt wird. Nicht selten wird die Enteignung als eine Option im Rahmen einer städtebaulichen Entwicklungsmaßnahme betrachtet (Schwer 1993; o. V. 2002a). Beispielsweise informiert die Frankfurter Rundschau über die Verlegung von Stromtrassen im Rahmen der Planungen eines neuen Stadtteils für bis zu 25.000 Menschen im Frankfurter Norden und über einen Brief, den die Eigentümer erhalten haben: „Mehr als 1000 Grundstückseigentümer haben jetzt Post von der Kommune bekommen. Sie sollen möglichst freiwillig ihren Grund und Boden an die Stadt veräußern. Geschieht das nicht, würde die von der Kommune eingeleitete städtebauliche Entwicklungsmaßnahme nach dem Baugesetzbuch am Ende auch Enteignungen ermöglichen" (o. V. 2018a). Hier fungiert die Zeitung nicht direkt als Plattform für eine Androhung, sondern als Informationsmedium, das über den Planungsprozess berichtet. In anderen Artikeln wird die Androhung einer Enteignung direkt von der entscheidenden Stelle ausgesprochen (o. V. 2002b; Schild 2012), beispielsweise vom Umweltminister Baden-Württembergs Franz Untersteller (Die Grünen) im Rahmen von Hochwasserschutzmaßnahmen: „Untersteller hofft zwar, dass ,am Ende immer die Vernunft siegt'. Zugleich unterstrich er: ,Wenn aber alle Versuche zu einer gütlichen Einigung erfolglos geblieben sind und es das Allgemeinwohl erfordert, muss als Ultima Ratio auch eine Enteignung möglich sein' (o. V. 2013).

Allerdings erfahren in 17 von 52 Artikeln (33\%), in denen die Möglichkeit einer Enteignung in Erwägung gezogen wird, die Leser/-innen noch im selben Artikel, dass die Wahrscheinlichkeit einer tatsächlichen Umsetzung niedrig ist. Beispielsweise berichtet die Frankfurter Rundschau bereits im Juni 2017 über die Planungen des neuen Wohnviertels im Norden Frankfurts und in diesem Zusammenhang über die Möglichkeit einer städtebaulichen Entwicklungsmaßnahme: „Etwa die Hälfte der gesamten Grundstücke befinden [sic] sich bereits im Besitz der Stadt oder stadtnaher Stiftungen. Doch nicht wenig Fläche gehört den örtlichen Landwirten. Die Bauern haben bereits das Ende ihrer Höfe vorausge- 
sagt, wenn sie Areale an den Wohnungsbau verlieren. Am Ende drohe innen die Enteignung, so etwa Wolfgang Stark, der Ortslandwirt von Praunheim. Genau dieser Befürchtung tritt Planungsdezernent Mike Josef entgegen. ,Es wird keine Enteignung geben', versichert Josefs Sprecher Gellert. Auch beim Stadtteil Riedberg habe sich die Kommune am Ende friedlich mit den Bauern einigen können. ,Es gab damals keine einzige Enteignung.“" (o. V. 2017b).

Auffallend an diesem Textausschnitt ist, dass zunächst die Betroffenen mit einer Enteignung rechnen und ein Entscheidungsträger diese Befürchtung relativiert. Zusammengefasst entsteht ein Bild, das die Ausgangshypothese der Drohkulisse nicht stützt: In gut einem Drittel der Artikel, die über die Erwägung einer Enteignung berichten, wird diese Androhung noch im Artikel widerlegt. Eine mögliche Drohwirkung verfällt also, noch bevor der Artikel zu Ende gelesen wurde. Im Text wird das Instrument zwar genannt, die Anwendung im selben Atemzug jedoch schon wieder ausgeschlossen: „Eine Enteignung von Land ist nach Angaben des Stadtplaners Leonhardt ,auf keinen Fall' vorgesehen" (Reinkowski 2004). Manchmal deutet sich schon in der Überschrift an, dass die Anwendung des Instruments sehr unwahrscheinlich ist. Die Stuttgarter Zeitung betitelt einen Artikel über eine geplante Städtebauliche Entwicklungsmaßnahme und mögliche Enteignungen mit „Zwang ist unwahrscheinlich" (Schieferecke 2018). Einerseits wird die Enteignung begrifflich ins Feld geführt: Als Botschaft bleibt damit zumindest auch hängen, dass die Anwendung möglich ist. Andererseits sei sie, so die Aussage im ersten Zitat, allerdings „auf keinen Fall“ vorgesehen, im zweiten Beispiel ist sie zumindest „unwahrscheinlich“.

Hinsichtlich der thematischen Schwerpunktsetzungen ist die Berichterstattung zur Enteignung etwas vielfältiger als die Berichterstattung zum Baugebot (siehe oben). In 77 der 141 Artikel (55\%) berichten die Zeitungen über Wohnen oder Gewerbeplanungen und in elf dieser 77 Artikel werden beide Themen gleichwertig behandelt. 26 Artikel (18\%) befassen sich mit einem Rechtsstreit. Insgesamt wird zwölfmal über verwahrloste Grundstücke und Bauruinen berichtet. Ansonsten ist die thematische Streuung deutlich größer als bei den Artikeln zum Baugebot, sodass insgesamt 47 Artikel mit dem Code "Sonstiges" markiert wurden. In diesen Artikeln wird beispielsweise über eine Enteignung im Zusammenhang mit einer Bundesgartenschau (Wagner 2001) oder Enteignungen im historischen Kontext (Stimmann 2009) berichtet.

In 32 von 141 Zeitungsartikeln (23\%) wird das sprachliche Gewicht, das mit dem Begriff Enteignung einhergeht, genutzt. In diesen Artikeln wird weder über eine vollzogene Enteignung berichtet noch wird mit der tatsächlichen Anwendung des Instruments gedroht. Stattdessen wird der Enteignungsbegriff benutzt, um die Eingriffsintensität eines anderen Instruments besonders stark zu betonen und zu kritisieren. Meistens erfolgt dies nicht durch Entscheidungsträger/-innen, sondern durch die Betroffenen oder die Opposition. Diese unterstellen dann, dass angekündigte Maßnahmen „an Enteignung grenzen" (o. V. 2004b) oder die Vorgehensweise der Verwaltung „einer Enteignung gleich“ komme (o. V. 2007; Werner 2009; o. V. 2011). Die Betroffenen verwenden den Begriff der "kalten Enteignung“ (Kerst 2002; o. V. 2009b; Neuber 2014). In einem Artikel aus der Stuttgarter Zeitung (o. V. 2014b) zu einem Rechtsstreit um die Rechtmäßigkeit eines Bebauungsplanes wird die Vorgehensweise als "Enteignung durch die Hintertür" bezeichnet. In anderen Fällen wird das Vorkaufsrecht als ein "enteignungsgleicher Eingriff" (Baumann 2015; o. V. 2017c) bezeichnet.

Die Analyse der 141 Zeitungsartikel zeigt, dass der Begriff Enteignung (einschließlich seiner negativen Konnotationen) Eigentümer nicht unbedingt einschüchtert, sondern sogar von innen verwendet wird, um die Rechtmäßigkeit kommunaler Planungen zu hinterfragen bzw. die Eingriffsintensität zu betonen. Wenn sich eine Gemeinde dem Verhältnismäßigkeitsprinzip folgend darum bemüht, ihre Planungen mit weniger eingriffsintensiven Instrumenten umzusetzen, dann kann durch die Verwendung des Begriffs „Enteignung“ durch die Betroffenen oder die Opposition bei einigen zeitunglesenden Menschen der Eindruck entstehen, die Maßnahme sei zu eingriffsintensiv und daher nicht verhältnismäßig. Des Weiteren lässt sich aus der Verwendung des Begriffs durch die Betroffenen ableiten, dass dem Begriff „Enteignung" eine Negativität unterstellt wird. Die Betroffenen setzen dieses sprachliche Gewicht diskursstrategisch ein.

\subsection{Kombination beider Instrumente}

Zum Abschluss der Ergebnisdiskussion erfolgt ein Blick auf die 44 Artikel, in denen beide Instrumente auftauchen. Neben den thematischen Schwerpunktsetzungen dieser Artikel (und ihren Unterschieden zu den anderen Artikeln) interessierte vor allem, in welchem Verhältnis die beiden Instrumente zueinander gesehen werden. Faktisch hat die Enteignung eine höhere Eingriffsintensität als das Baugebot und ist auch am Ende eines Baugebotsverfahrens als ,ultima ratio' zumindest theoretisch möglich (§ 176 Abs. 8 BauGB). Inhaltlich geht es in 
diesen Artikeln hauptsächlich um Wohnnutzung (17 von 44 Artikeln, $39 \%$ ) und Bauruinen (21 Artikel, 48 \%). Die Artikel ähneln stark den Artikeln, die sich ausschließlich mit dem Baugebot beschäftigen - mit dem Unterschied, dass zusätzlich die Enteignung genannt wird. Zitate wie das folgende sind allerdings auch in dieser Gruppe eher die Ausnahme: „Im Abendblatt-Gespräch forderte der Grünen-Politiker: ,Wenn der Eigentümer weiterhin das Grundstück offensichtlich als Spekulationsobjekt missbraucht und hier nichts passiert, dann muss ein Baugebot oder als letzte Lösung sogar eine Enteignung her."“ (Gaßdorf 2014).

Eher schon attestieren die Autorinnen bzw. Autoren und die zitierten Personen beiden Instrumenten gleichzeitig eine geringe Umsetzungswahrscheinlichkeit, wie in diesem Fall durch Rheinhausens Bezirksamtsleiter Reiner Sanner: „Wenn alles nichts hilft, gäbe es theoretisch - aber wirklich nur theoretisch - noch drastische Maßnahmen wie Enteignungen oder ein so genanntes ,Baugebot', das zur Bebauung verpflichtet. Derlei Schritte lassen sich aber so gut wie nie durchsetzen, so Sanner: ,Das sind äußerst seltene Instrumente, und es gibt dagegen jede Menge Interventionsmöglichkeiten. Das würde sich ewig hinziehen."“ (Zehrfeld 2011).

Bemerkenswert ist ein Artikel, in welchem die Forderung eines Baugebots nicht durch die Kommune, sondern von den Bürgerinnen und Bürgern erhoben wird: „Morgen, Donnerstag, kommt die Bezirksvertretung III zusammen. Dann steht auf der Tagesordnung der Bürgerantrag zur Brandruine, in der früher das Lokal ,Alt Schlebusch' ansässig war. Darin fordern die Bürger ein Baugebot - heißt: Die Gemeinde kann den Eigentümer etwa verpflichten, sein Grundstück zu bebauen oder ein vorhandenes Gebäude den Festsetzungen des Bebauungsplans anzupassen (wir berichteten). Die Stadt hat nun ihre Stellungnahme abgegeben. Nach Prüfung - und Ortstermin mit Eigentümer und Sachverständigem, aus dessen Gutachten die Stadt berichten will - rät sie vom Baugebot ab. [Eine] Enteignung käme ,nur in Betracht, wenn der Eigentümer der Verpflichtung aus dem Baugebot nicht nachkommt'. Falls dieses ausgesprochen werde, würde die Stadt bei einem Klageverfahren ,höchstwahrscheinlich unterliegen' und müsste die Kosten tragen, sagt sie" (o.V. 2018b). Die Kommune übernimmt in diesem Textausschnitt die Rolle der Experten/Expertinnen, die die Bürgerschaft darüber aufklärt, warum die Umsetzung beider Instrumente unwahrscheinlich ist. Auch wenn solche Textausschnitte eher die Ausnahme darstellen, stehen sie im Kontrast sowohl zu der Ausgangshypothese als auch zu dem Diskurs um Boris Palmer aus Tübingen.

\section{Zur Wirksamkeit der Responsivität}

Die Analyse der Zeitungsmedien liefert zunächst einmal ein weiteres Indiz, dass tatsächliche Anwendungsfälle der beiden untersuchten Instrumente selten sind. Im untersuchten Material finden sich einige Fälle, in denen von einer tatsächlichen Anwendung sowohl des Baugebots als auch der Enteignung gesprochen wird. Die Anwendungen des Baugebots entpuppen sich als privatrechtliche Vereinbarungen, die vertraglich eine Frist zur Überbauung vorsehen. Der Begriff „Enteignung“ wird hingegen unter anderem dazu verwendet, die Unverhältnismäßigkeit anderer Instrumente zu kritisieren, die rechtswissenschaftlich nicht als Enteignung, sondern höchstens als Inhalts- und Schrankenbestimmungen des Eigentums anzusehen sind.

Die Theorie der responsiven Bodenpolitik besagt, dass eingriffsärmere Instrumente deshalb wirken, weil sich alle Beteiligten und insbesondere die Betroffenen der Existenz eingriffsintensiverer Instrumente bewusst sind. So könnten Planungsträger eine Planumsetzung auch ohne eine konkrete Anwendung eingriffsintensiver Instrumente erwirken. Die Anwendung weniger eingriffsintensiver Instrumente ist schon deshalb die Regel, weil Eigentümer rational handeln und von der Planumsetzung - vor allem ökonomisch - profitieren. Zeigen sich Eigentümer, aus welchen Gründen auch immer, nicht kooperationsbereit, sollen, so die Theorie, auch dann die milden Instrumente wirken, allerdings aus einem anderen Grund. Wenn Eigentümer nicht kooperieren, müssen sie mit eingriffsintensiven Instrumenten rechnen. Der vorliegende Beitrag hat untersucht, ob und wie diese Möglichkeit durch Ämter und Gemeinden auf medialer Ebene kommuniziert wird.

Im untersuchten öffentlichen Diskurs zeigt sich, dass sich die betroffenen Grundstückseigentümer häufig nicht einschüchtern lassen, was auch auf eine differenzierende Berichterstattung durch meist gut informierte Autorinnen und Autoren zurückzuführen ist (ungeachtet der oben genannten gelegentlichen begrifflichen Ungenauigkeiten in der Berichterstattung zum Baugebot). Im Ergebnis zeugt dies von geringen Hemmungen der Eigentümer, eine allfällige juristische Auseinandersetzung in Kauf zu nehmen. Darüber hinaus zeigt sich sogar, dass nicht nur die öffentlichen Planungsträger, sondern auch die Betroffenen diskursstrategisch handeln können. Sie setzen die Nennung der Instrumente gezielt ein, um gegen ungeliebte Planungsmaßnahmen anzugehen. 
Der vorliegende Beitrag erlaubt Erkenntnisse, die über die sonst üblichen rechts- oder planungswissenschaftlichen Möglichkeiten von Urteilsanalysen und Fallbeispieldarstellungen hinausgehen. Jedoch ist auch dieser Ansatz Limitierungen unterworfen. So ist nicht auszuschließen, dass die entscheidenden Gespräche und Verhandlungen (einschließlich möglicher Androhungen) zwischen den Akteuren außerhalb der medialen Öffentlichkeit stattfinden. Gleichwohl, der Titel des vorliegenden Artikels nimmt das wichtigste Ergebnis vorweg: Die Hypothese, dass das Baugebot und die Enteignung von öffentlichen Planungsträgern in lokalen und regionalen Medien diskursstrategisch erfolgreich verwendet wird, um Eigentümer zur Kooperation zu veranlassen, lässt sich anhand der Untersuchung nicht eindeutig bestätigen. Die in der Planungswissenschaft zugesprochene responsive Wirkung gilt bei kooperationsunwilligen, aber aufgeklärten Eigentümern nicht.

\section{Literatur}

Antoniazza, Y. (2008): Die Baupflicht. Zürich.

Bischoff, A.; Selle, K.; Sinning, H. (2007): Informieren, Beteiligen, Kooperieren - Kommunikation in Planungsprozessen. Eine Übersicht zu Formen, Verfahren und Methoden. Dortmund.

BMI - Bundesministerium des Innern, für Bau und Heimat (2019): Empfehlungen auf Grundlage der Beratungen in der Kommission für „Nachhaltige Baulandmobilisierung und Bodenpolitik" (Baulandkommission) vom 2. Juli 2019. Berlin.

Davy, B. (2005): Grundstückswerte, Stadtumbau und Bodenpolitik. In: vhw Forum Wohneigentum 2, 67-72.

Davy, B. (2006): Innovationspotenziale für Flächenentwicklung in schrumpfenden Städten. Wissenschaftliche Studie im Auftrag erstellt für die Internationale Bauausstellung Stadtumbau Sachsen-Anhalt 2010. Magdeburg.

Dieterich, H. (1983): Baugebot - ein Weg zur Bebauung erschlossener Baugrundstücke? In: Städte- und Gemeindebund 38, 2, 43-49.

Dieterich, H. (1985): Baulandumlegung - Recht und Praxis. München.

Flyvbjerg, B. (1998): Rationality and Power: Democracy in Practice. London.

Forester, J. (1989): Planning in the Face of Power. London.

Gerber, J.-D.; Knoepfel, P.; Nahrath, S.; Varone, F. (2009): Institutional Resource Regimes: Towards sustainability through the combination of property-rights theory and policy analysis. In: Ecological Economics 68, 3, 798-809. doi: 10.1016/j.ecolecon.2008.06.013

Günzel, M. (2016): „Was im Dunkeln liegt, kann man nicht sehen“Eine Spurensuche nach diskurstheoretischen Impulsen in der Raumplanung. In: pnd online 1, 1-10.

Healey, P. (1992): Planning Through Debate. The Communicative Turn in Planning Theory. In: Town Planning Review 63, 2, 143162. doi: $10.2307 / 40113141$
Hengstermann, A. (2017): Baugebot und Bauverpflichtung - Vergleich der Regelungen im deutschsprachigen Planungsrecht. In: Flächenmanagement und Bodenordnung 79, 4, 153-159.

Hengstermann, A.; Gerber, J.-D. (2015): Aktive Bodenpolitik - Eine Auseinandersetzung vor dem Hintergrund der Revision des eidgenössischen Raumplanungsgesetzes. In: Flächenmanagement und Bodenordnung 77, 6, 241-250.

Hengstermann, A.; Gerber, J.-D. (2017): Die faktische und explizite Bauverpflichtung - eine Rezension des bodenpolitischen Instruments in Recht und Praxis. In: Umwelt- und Planungsrecht 37, 1, 16-24.

Hengstermann, A.; Hartmann, T. (2018): Instruments of Land Policy: Four Types of Intervention. In: Gerber, J.-D.; Hartmann, T.; Hengstermann, A. (Hrsg.): Instruments of Land Policy. Dealing with Scarcity of Land. London, 27-32.

Keller, R. (2011): Diskursforschung. Eine Einführung für Sozialwissenschaftlerlnnen. Wiesbaden. doi: 10.1007/978-3531-92085-6

Köhler, H. (1985): Die Planverwirklichungsgebote als Instrument des Städtebaurechts. Göttingen. = Schriftenreihe des Deutschen Städte- und Gemeindebundes 44.

Kolocek, M. (2009): Der Shopping Center Diskurs. Unveröffentlichte Diplomarbeit an der Fakultät Raumplanung der Technischen Universität Dortmund.

Kolocek, M. (2014): The Human Right to Housing: Using ATLAS. ti to Combine Qualitative and Quantitative Methods to Analyse Global Discourses. In: Friese, S.; Ringmayr, T. G. (Hrsg.): ATLAS.ti User Conference 2013: Fostering Dialog on Qualitative Methods. Berlin, 1-13.

Kolocek, M. (2017): The Human Right to Housing in the Face of Land Policy and Social Citizenship. A Global Discourse Analysis. Basingstoke. doi: 10.1007/978-3-319-53489-3

Kolocek, M. (2018): A German Perspective on Building Obligations: Planning Professionals Try to Remember. In: Gerber, J.-D.; Hartmann, T.; Hengstermann, A. (Hrsg.): Instruments of Land Policy. Dealing with Scarcity of Land. London, 189-192.

Lege, J. (2011): Das Eigentumsgrundrecht aus Art. 14 GG. Teil 1: Verfassungsrechtliche Grundlagen. In: JURA - Juristische Ausbildung 33, 7, 507-519. doi: 10.1515/JURA.2011.099

Leisner, R. (2009): Baugebot und Baufreiheit: Das Spannungsverhältnis zwischen dem einfach-gesetzlich ausgestalteten Baugebot und der verfassungsrechtlichen Garantie des Eigentums. Berlin. = Schriften zum öffentlichen Recht 1126.

Lobeck, M.; Wiegandt, C.; Wiese-von Ofen, I. (2006): Entwicklung von umsetzungsorientierten Handlungsschritten zur Mobilisierung von Baulücken und zur Erleichterung von Nutzungsänderungen im Bestand in Innenstädten NRWs. Bonn.

Lücke, J. (1980): Das Baugebot - Ein wirksames Instrument des Bodenrechts? München.

Müller, G. (1989): Baupflicht und Eigentumsordnung. In: Haller, W.; Kölz, A.; Müller, G.; Thürer, D. (Hrsg.): Festschrift für Ulrich Häfelin zum 65. Geburtstag. Zürich, 167-181.

Prantl, H. (2019): Eigentum verpflichtet. Das unerfüllte Grundgesetz. München.

Reuter, W. (2000): Zur Komplementarität von Diskurs und Macht in der Planung. In: disp - The Town Planning Review 36, 141, 4-16. doi: 10.1080/02513625.2000.10556733 
Saladin, P. (1982): Grundrechte im Wandel - Die Rechtsprechung des Schweizerischen Bundesgerichts zu den Grundrechten in einer sich ändernden Umwelt. Bern.

Schäfer, R. (1983): Planverwirklichungsgebote in der kommunalen Praxis. Berlin.

Schlick, W. (2018): „Nassauskiesung“ und ihre Folgen Wirkungsgeschichte einer Entscheidung. In: Depenheuer, O.; Shirvani, F. (Hrsg.): Die Enteignung. Historische, vergleichende, dogmatische und politische Perspektiven auf ein Rechtsinstitut. Berlin, 111-136. doi: 10.1007/978-3-66254690-1_5

Schlichter, O. (1993): Überlegungen zum Baugebot. In: Driehaus, H.-J.; Birk, H.-J. (Hrsg.): Baurecht Aktuell - Festschrift für Felix Weyreuther. Köln, 350-370.

Schmidt-Eichstaedt, G. (2005): Städtebaurecht. Berlin.

Schwab-Trapp, M. (2001): Diskurs als soziologisches Konzept. Bausteine für eine soziologisch orientierte Diskursanalyse. In: Keller, R.; Hirseland, A.; Schneider, W.; Viehöver, W. (Hrsg.): Handbuch Sozialwissenschaftliche Diskursanalyse. Band 1: Theorien und Methoden. Wiesbaden, 263-285.

Schwab-Trapp, M. (2003): MethodischeAspekte derDiskursanalyse. Probleme der Analyse diskursiver Auseinandersetzungen am Beispiel der deutschen Diskussion über den Kosovokrieg. In: Keller, R.; Hirseland, A.; Schneider, W.; Viehöver, W. (Hrsg.): Handbuch Sozialwissenschaftliche Diskursanalyse. Band 2: Forschungspraxis. Wiesbaden, 171-196. doi: 10.1007/978-3322-99674-9 7

Selle, K. (2013): Über Bürgerbeteiligung hinaus: Stadtentwicklung als Gemeinschaftsaufgabe? Analysen und Konzepte. Detmold.

Stüer, B. (1988): Baugebot nach § 176 BauGB. In: Die Öffentliche Verwaltung 41, 8, 337-340.

Thiel, F. (2019): Art. 15 Grundgesetz - obsolet? Helmut Ridder zum 100. Geburtstag. In: Die Öffentliche Verwaltung 72, 13, 497507.

Viallon, F.-X.; Schweizer, R.; Varone, F. (2019): When the Regime Goes Local: Local Regulatory Arrangements and Land Use Sustainability. In: Environmental Science and Policy 96, 77-84. doi: 10.1016/j.envsci.2019.02.010

Vogel, H.-J. (2019): Mehr Gerechtigkeit! Wir brauchen eine neue Bodenordnung - nur dann wird auch Wohnen wieder bezahlbar. Freiburg im Breisgau.

Wissenschaftliche Dienste des Bundestags (2018): Sachstand. Rechtsprechung zum Baugebot nach $\S 176$ BauGB. Az.: WD $7-3000-065 / 18$. Berlin

\section{Zitierte Artikel}

Baumann, A. (2015): Konflikt um Kurfürsten-Gelände entschärft. Stadtverwaltung und Eigentümer einigen sich auf gemeinsames Vorgehen beim Bebauungsplanverfahren. In: General-Anzeiger vom 6. März 2015.

Biermann, R. (2007): Baustopp am ehemaligen Kaufhof dauert an. Auf dem Gelände an der Leipziger Straße in Bockenheim stagniert die Arbeit, weil Nachweise für Brandschutz und Statik weiterhin fehlen. In: Frankfurter Rundschau vom 16. Februar 2007.

Gaßdorf, U. (2014): Enteignung als „letzte Lösung“. In: Die Welt (Hamburg) vom 4. August 2014.
Göpfert, C.-J. (2003): Kindermann kündigt Vertrag für Rekonstruktion der Gerbermühle. Architekt Albert Speer ist „sehr verärgert“, die Planer im Römer ratlos. Hoffnung auf neuen, zuverlässigen Investor. In: Frankfurter Rundschau vom 6. Januar 2003.

Goethals, G. (2007): Bürger stören sich an Schandflecken. Gießener verlangen Sanierung des Samen-Hahn-Gebäudes. Manche Grundstücke liegen seit vielen Jahren brach. In: Frankfurter Rundschau vom 24. Januar 2007.

Goltze, E. (2011): Städtische Bauplätze werden knapp. Interesse ist groß. In: Gießener Anzeiger vom 6. August 2011.

Hille, C. O. (2000): Dieser Schandfleck muss verschwinden. In: Kölner Express vom 7. Juni 2000.

Kerst, M. (2002): So platzt der Olympia-Traum. Widerstände von allen Seiten. In: Kölner Express vom 22. März 2002.

Lauchs, G. (1994): 14 Grundstückseigentümer strengten gegen drohende Enteignung Normenkontrollklage an. In: Nürnberger Nachrichten vom 23. November 1994.

Neuber, R. (2014): „Kommt kalter Enteignung gleich“. Verein für gerechte Kommunalabgaben zweifelt an Begründung für Ausbau der Mühlenstraße. In: Allgemeine Zeitung vom 7. Januar 2014.

o. V. (2000): Mehr Mut zur Baulücke. In: taz vom 9. Dezember 2000. o. V. (2001). Längste Baupause am Kurfürstendamm geht zu Ende. In: Die Welt vom 29. Dezember 2001.

o. V. (2002a): Für den Wohnpark kann die Gemeinde enteignen. Ergebnis der Städtebaulichen Voruntersuchung für den Wohnpark Grafschaft in Ringen wird im Herbst erwartet. In: General-Anzeiger vom 13. April 2002.

o. V. (2002b): Einstimmiges Votum gegen den „Wohnpark Grafschaft". Vorschlag: Mehrere Baugebiete ausweisen. In: General-Anzeiger vom 27. April 2002.

o. V. (2004a): City-West. Warten auf Durchbruch. In: ImmobilienZeitung vom 12. Februar 2004.

o. V. (2004b): Kein Häuserkampf am Schwarzen Meer. Der Landesdenkmalschützer in der Debatte um den Erhalt der historischen Gebäude des Klinikums Mitte tritt als Kompromissler auf. In: taz vom 6. Dezember 2004.

o. V. (2007): Mut zur Lücke oder Enteignung? Stadtumbau Ost. „Die Leute ziehen nicht in die Altbausubstanz“. In: ImmobilienZeitung vom 28. Juni 2007.

o. V. (2009a): Noch viel Bauland übrig. Stadt bereitet Ende der Entwicklungsmaßnahme vor. In: Frankfurter Rundschau vom 17. Februar 2009.

o. V. (2009b): Projekt Rheinblick: Ein Partner wirft Stadt „kalte Enteignung" vor. In: Rheinische Post vom 7. April 2009.

o. V. (2011): Wenn Immobilien verfallen. In: Stuttgarter Zeitung vom 26. November 2011.

o. V. (2013): Enteignungen nicht tabu. Umweltminister Untersteller will besseren Schutz gegen Hochwasser durchsetzen. In: Südwest-Presse vom 15. Juli 2013.

o. V. (2014a): „Wirtschaftlich darstellbar“. Gutachterausschuss prüft Wohnnutzung. In: Gießener Anzeiger vom 5. November 2014.

o. V. (2014b): Enteignung durch die Hintertür? In: Stuttgarter Zeitung vom 3. Februar 2014.

o. V. (2015): Stadt hat nur ,,ein stumpfes Schwert“. Zwang hilft beim Kaufhof nicht. In: Nürnberger Nachrichten vom 17. März 2015.

o. V. (2016): Launsbacher Baugebiet stand kurz auf der Kippe. Ministerium hatte Probleme wegen Dorfentwicklung. Lösung in Sicht. In: Gießener Anzeiger vom 11. Juni 2016. 
o. V. (2017a): Der Druck wächst. In: Frankfurter Rundschau vom 9. Dezember 2017.

o. V. (2017b): Zehn Jahre bis zum ersten Wohnhaus. In: Frankfurter Rundschau vom 17. Juni 2017.

o. V. (2017c): Negativzeugnis ausgestellt. Grundstücksstreit - Stadt Sulz übt ein Vorkaufsrecht an der Balinger Straße nicht aus. In: Schwarzwälder Bote vom 28. Oktober 2017.

o. V. (2018a): Stromtrassen sollen unter die Erde. In: Frankfurter Rundschau vom 3. Juli 2018.

o. V. (2018b): Brandruine: Stadt rät von Baugebot ab. In: Rheinische Post vom 27. Juni 2018.

o. V. (2019): Boris Palmer will Eigentümer zum Bauen zwingen. In: Tagesspiegel vom 27. April 2019.

Puderbach, J. (2012): Politik hat kein Mittel gegen Bauruinen. Weiterbau oder Abriss können nicht erzwungen werden. In: Kölner Stadt-Anzeiger vom 11. Dezember 2012.

Reinkowski, M. (2004): Beim Grunderwerb geht es um alles oder nichts. Bevor Daimler den Bauantrag zur Erweiterung des Technologiezentrums stellen kann, muss die Firma im Besitz der Flächen sein. In: Stuttgarter Zeitung vom 12. Oktober 2004.

Schieferecke, M. (2018): Verwaltung könnte gar zu Enteignung greifen. In: Stuttgarter Zeitung vom 30. November 2018.

Schild, H. (2012): Voerde führt Wohnbaulandkonzept ein. In: Rheinische Post vom 5. Juli 2012.

Schwarz, K. (2005): Streit um neue Wohnbauflächen entbrannt. Finanzbürgermeister will 100 Hektar auch außerhalb des Flächennutzungsplans erschließen - SPD warnt. In: Stuttgarter Nachrichten vom 24. August 2005.

Schwer, M. (1993): Mit städtebaulicher Entwicklungsmaßnahme können Kommunen Bodenpreise kontrollieren. Wirksames Mittel gegen Spekulation. Wertzuwachs kommt der Gemeinde zugute - Im Streitfall Enteignung. In: Nürnberger Nachrichten vom 27. März 1993.

Stiefelhagen, S. (2011): Die Fußgängerzone soll bleiben. Zu Plänen für die Schlossstraße und zum ersten Architektenvorschlag. In: Kölner Stadt-Anzeiger vom 26. März 2011.

Stimmann, H. (2009): Stadt ohne Altstadt. Berlins historische Mitte: Warum die Metropole die Leere zwischen Schloss und Alexanderplatz wieder bebauen sollte - Ein Plädoyer. In: Die Welt vom 11. Mai 2009.

von Appen, K. (1994): Neubau-Gebot. In: taz vom 19. Februar 1994 Wagner, L. (2001): Folgenreicher Streit um eine Enteignung für die Gartenschau; Bewertung von Grundstücken seit 1991 unklar. In: Die Welt vom 14. Juli 2001.

Werner, J. (2009): Dinslaken will's zügig. In: Rheinische Post vom 17. März 2009.

Wolf, T. (2016): Wem gehört Ihr? In: Sächsische Zeitung vom 17. September 2016.

Zehrfeld, S. (2011): Neues Viertel geplant auf altem Schulgelände. In: Rheinische Post vom 13. September 2011. 